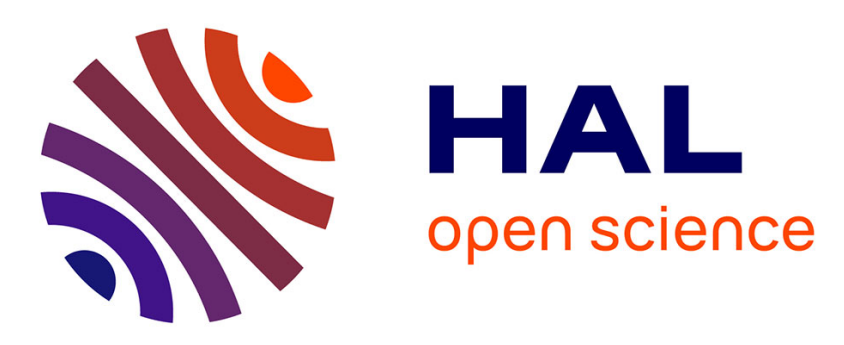

\title{
A closed loop musculoskeletal model of postural coordination dynamics
}

\author{
Vincent Bonnet, Philippe Fraisse, Nacim Ramdani, Julien Lagarde, Sofiane \\ Ramdani, Benoit G. Bardy
}

\section{- To cite this version:}

Vincent Bonnet, Philippe Fraisse, Nacim Ramdani, Julien Lagarde, Sofiane Ramdani, et al.. A closed loop musculoskeletal model of postural coordination dynamics. CDC/CCC'09: 48th IEEE Conference on Decision and Control, held jointly with the 28th Chinese Control Conference, Dec 2009, Shanghai, China. pp.6207-6212, 10.1109/CDC.2009.5400945 . lirmm-00798599

\section{HAL Id: lirmm-00798599 \\ https://hal-lirmm.ccsd.cnrs.fr/lirmm-00798599}

Submitted on 8 Mar 2013

HAL is a multi-disciplinary open access archive for the deposit and dissemination of scientific research documents, whether they are published or not. The documents may come from teaching and research institutions in France or abroad, or from public or private research centers.
L'archive ouverte pluridisciplinaire HAL, est destinée au dépôt et à la diffusion de documents scientifiques de niveau recherche, publiés ou non, émanant des établissements d'enseignement et de recherche français ou étrangers, des laboratoires publics ou privés. 


\title{
A closed loop musculoskeletal model of postural coordination dynamics
}

\author{
Vincent Bonnet, Philippe Fraisse, Nacim Ramdani, Julien Lagarde, Sofiane Ramdani, Benoit G. Bardy
}

\begin{abstract}
A closed-loop model with actuator dynamics and sensory feedback has been developed to capture the complex postural behaviors observed in a human head tracking task. In motor-control litterature, spindle feedback gains are scaled by the central nervous system to adapt muscle stiffness depending on the postural task. We propose to identify spindle reflex equivalent feedback gains for several target's frequency values. Comparison with experimental results on human shows the relevance of this modeling, since our musculoskeletal model is able to exhibit reasonably well the behavioral invariants observed in human postures.
\end{abstract}

Index Terms-Human Postural Coordination, Dynamical Postural controller, Redundant Systems, Biological system modeling, Parameter estimation.

\section{INTRODUCTION}

The coordination and control of human posture (the spatiotemporal organization of body joints) and balance have been modelled in several ways using many experimental paradigms, in tasks involving postural responses to external perturbations or to intentional tracking tasks.

During a visual tracking task, Bardy et al [1] analyzed, in the framework of coordination dynamics [2], the joint coordination in the sagittal plane. They proposed the use of a collective variable to describe in a simple way the complex dynamical biological-segmental-articular-muscularcouplings. They had standing participants moving back and forth in the sagittal plane in order to track the displacement of a virtual target. This simple task allowed the observation of several self-organized properties of the postural system, such as phase transition, multistability, critical fluctuations, hysteresis, and critical slowing down. The collective variable able to capture both fully and in a very compact way these dynamical properties, is the relative phase, i.e. the phase difference between the ankle and the hip. Two coordination modes were observed between ankle and hip depending on the target's frequency: An in-phase mode for low frequencies and an anti-phase mode for high frequencies.

Martin et al. [3] used a constrained optimization process to analyze Bardy et al.'s [1] results, and showed that the location of the center of pressure $(\mathrm{CoP})$ can drive the selection of the coordination mode. In a previous work [4], we studied in more details Bardy et al.'s [1] paradigm with

V. Bonnet, P. Fraisse and N. Ramdani are with the LIRMM UMR 5506 CNRS, Univ. Montpellier 2, 161 rue Ada, Montpellier, 34392 France.

N. Ramdani is with the INRIA Sophia-Antipolis, Nice FR-06902, France.

J. Lagarde, S. Ramdani and B. G. Bardy are with the EDM EA 2991,

Univ. Montpellier 1, EDM, 700 av. du pic Saint Loup, Montpellier, 34090 France.

B. G. Bardy is with Institut Universitaire de France, 103 Bd St Michel, Paris, 75005 France a method similar to the one used in [3]. Then we implemented the obtained coordination modes onto the HOAP-3 and HRP2 humanoid robots. We showed that the in-phase mode corresponds to the minimum energy mode for low frequencies, and that the anti-phase mode is the only one able to maintain balance for high frequencies.

However, the approach described above considers only steady state behaviors and thus is not capable of capturing the transient dynamics observed during human postural behaviors such as the hysteresis phenomenon for instance. Nonlinear coupled oscillators are classically used to model these human dynamical coupling phenomena [5]. However, these oscillators involve several unknown parameters which have to be tuned and whose connection with the actual system is difficult to delineate.

In tasks involving postural responses to external perturbations, Nasher and Mc Collum [6] observed two postural strategies at the muscular activation level. One of two postural strategies are adopted according to perturbation magnitude: either a large muscular activation level at the ankles, or a coordinated activation of the hips and the ankles. Hemami [7] and colleagues have found that reasonable predictions behaviour can be made using linearized dynamics to model small perturbations postural response. Based on these postural strategies and assumptions, Kuo [8] developed a double inverted pendulum (DIP) model with an optimal control law weighting excursion of the center of mass and deviations from the upright position, and with feasible acceleration set (FAS) which is connected to the physiological limitations and equilibrium constraint. Using the FAS framework, Park et al. [9] optimized joint feedback gains to fit human data for different kind of perturbations and showed that the trajectories of joint angles and joint torques scale with perturbation magnitude in agreement with the postural strategy observations. In the same way, Jo et al. [10] proposed a cerebrocerebello-spinomuscular model of human posture with time delays and proportional-integrativederivative feedbacks ; their controller uses different set of cerebellum gains depending on perturbation magnitude.

These modeling approaches have inspired our work in the postural coordination framework. In this paper, we report the non-linear closed loop model we are developing in order to capture and predict postural sway movements during head tracking tasks. Our model is composed of a double inverted pendulum as biomechanical model, muscles models at each joint and a classical proportional-derivative controller in the operational space. In this sequel, we will report human experimentations first, in order to describe the postural coordination concept, and then we will describe 
the modelling and the identification of model parameters in subsequent sections.

\section{HUMAN EXPERIMENTATION}

The aim of the experiment is to provide a database of human behavior in order to identify spindle feedback gains in section IV.

\section{A. Methods}

Following previous studies [11], [12], the experiment consists in tracking a moving target with the head while standing. Participants stood on a force platform in front of a physical target moved by a linear motor in antero-posterior direction, with the knees locked and the soles constantly in contact with the ground (Fig. 1-2-3).

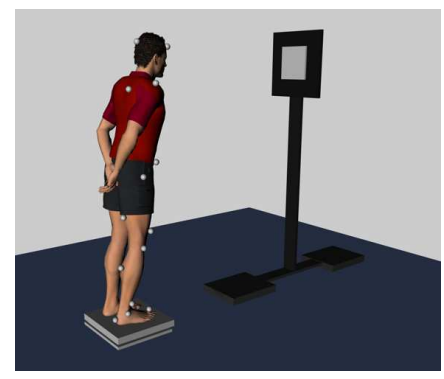

Fig. 1. Experimental device. Physical target moved by a linear motor, force plate and motion capture device.

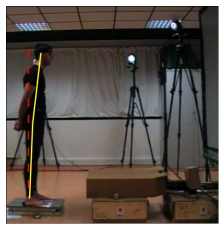

(a) $\mathrm{t}=0 \mathrm{sec}$

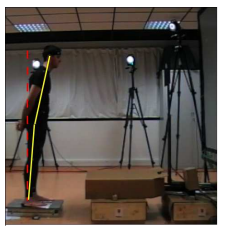

(b) $\mathrm{t}=1 \mathrm{sec}$

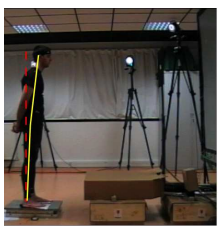

(c) $\mathrm{t}=2.5 \mathrm{sec}$
Fig. 2. Human typical experiments at $0.2 \mathrm{~Hz}$. Coordinative in-phase small displacement of the ankle and the hip.

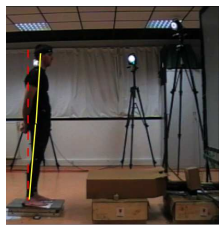

(a) $\mathrm{t}=0 \mathrm{sec}$

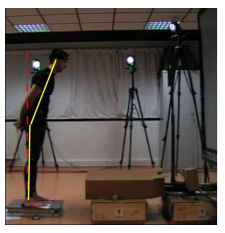

(b) $\mathrm{t}=0.5 \mathrm{sec}$

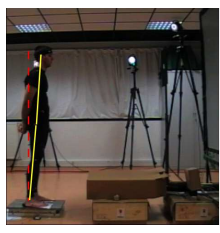

(c) $\mathrm{t}=1.3 \mathrm{sec}$
Fig. 3. Human typical experiments at $0.6 \mathrm{~Hz}$. Coordinative anti-phase displacement of the ankle and the hip. The hip amplitude is larger than the ankle one.

The experiment was performed on 11 healthy male subjects, with mean age 25 , mean weight $75 \mathrm{~kg}$ and mean size $1.79 \mathrm{~m}$. Target motion was sinusoidal with $10 \mathrm{~cm}$ as amplitude, the frequency increases from $0.1 \mathrm{~Hz}$ to $0.65 \mathrm{~Hz}$ by $0.05 \mathrm{~Hz}$ steps and during 10 periods. To capture the joint positions, a motion capture system (VICON NEXUS) was used, with 8 cameras (MX13) tracking 15 makers on the right side of the subject.
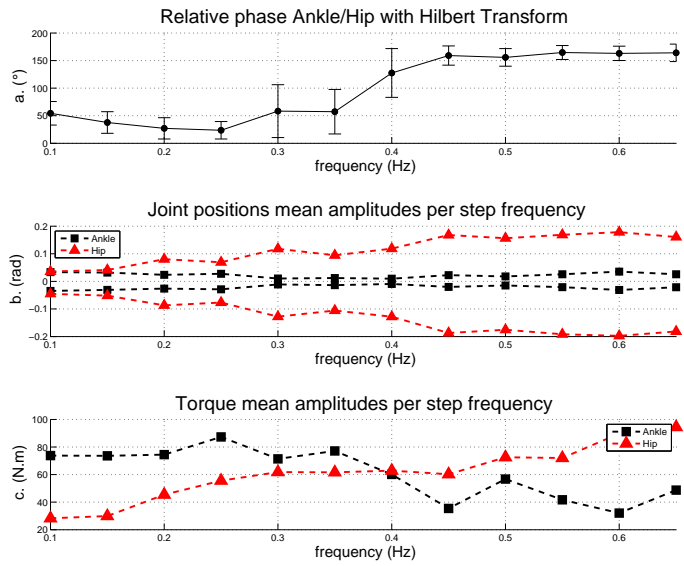

Fig. 4. Typical human experimental results. (a) Ankle/hip relative phase, showing a transition frequency around $0.4 \mathrm{~Hz}$ (b) Peak to peak joint positions. (c) Estimation of joint torque amplitudes.

\section{B. Experimental results}

Fig.4 shows typical results for a representative subject (weight $75 \mathrm{~kg}$, size $1.80 \mathrm{~m}$ ). On Fig. $4 \mathrm{a}$, the mean values of the relative phase (Hilbert-transformed) between ankle and hip positions are represented as a function of the frequency step. The depicted error bars correspond to the standard deviations during the 10 oscillations achieved at each frequency step. A transition is observed from in-phase to anti-phase mode around $0.4 \mathrm{~Hz}$. Joint positions are presented on Fig. $4 \mathrm{~b}$ by minima and maxima values. Each point is the mean value of the maximum (or minimum) joint position reached during the 10 oscillation periods performed at each frequency step. For the in-phase mode, i.e., at low frequencies, the joint positions amplitude difference are small, with individual differences in terms of joint amplitude. At the transition frequency, the ankle amplitude become very small (Fig. 10), and the relative phase between ankle and hip is difficult to estimate. This strong reduction of the ankle amplitude is typical of human phase transition [11], [12], [13]. The hip amplitude is larger than the ankle amplitude for the anti-phase mode as mentioned in [11], [12], [13]. Fig. 4(c) depicts mean values for torque amplitude estimation at each frequency step. Torque values were estimated by using the inverse dynamical model of the DIP. They indicate a larger ankle torque amplitude for in-phase mode and a larger hip torque amplitude for anti-phase in agreement with the ankle and hip strategy reported in [6] and by Runge et al. [14].

These observations hold for all participants and are in accordance with [11], [1], [13], even though the actual transition frequency and joint amplitudes depend on the specific subject body type.

\section{Modeling POSTURAL BeHAVIOR}

The model of postural coordination consists of 4 major components: the rigid body mechanics, the muscle dynamics, the reflex feedback loop and the closed-loop controller. 


\section{A. Biomechanical model}

Barin [15] shows the relevance of an inverted pendulum structure in the case of a human sagittal plane task. In addition, the Bardy's paradigm focused on the hip and ankle joints, so a DIP in the sagittal plane is used in this paper as a biomechanical model (Fig. 5).

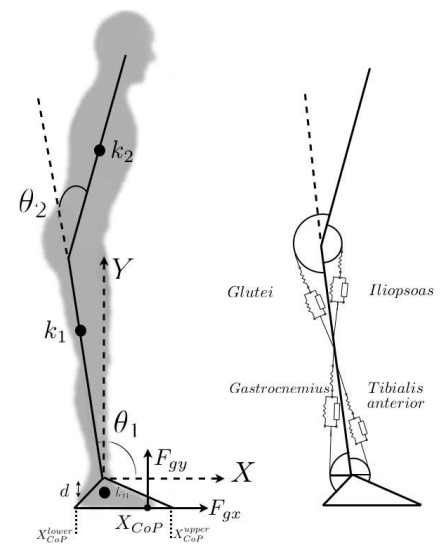

Fig. 5. Double inverted pendulum used to model postural coordination and a musculoskeletal model. The moment arms of muscular forces are the pulleys radius.

Balance is described by the position of the CoP within the $\mathrm{BoS}$, which can be expressed as a function of the dynamic parameters (eq.1).

$$
X_{C o P}=\left(\Gamma_{1}-F_{g x} d+m_{0} k_{0} g\right) / F_{g y}
$$

where $F_{g x}$ is the horizontal ground reaction force, $F_{g y}$ the vertical one, $\Gamma_{1}$ the ankle torque, and $m_{0}$ and $k_{0}$ foot parameters. Euler's equations were used for the calculation of the ground reaction forces as proposed by Cahouet et al. [16]. The muscle length evolutions, function of the joint positions, are computed by using a double pulley model at each joint (see Fig. 5). Muscle insertion points and parameters are adapted from [17].

\section{B. Joint actuator dynamics}

Musculotendon contraction dynamics is often modeled with Hill type models. For postural applications, Hill type models are often linearized [18], but in our application, we will keep the non-linear dynamic phenomenon. Furthermore many musculoskeletal models as [19] consider the tendon completely stiff. To provide a realistic model, the compliant tendon in the model needs to be addressed because of the long ankle tendons. Then a 3 element Hill-type model (Fig. 6) is used, based on [20]. The muscle model is composed of an active contractile element (CE) a passive parallel element (PE), which represents the intrinsic viscoelastic muscle properties, and a serial linear tendon (SE). Normalized units are used in this model to produce the output force $F_{M t}$, which results of the interaction between the force produced by muscle and the tendon stiffness, i.e. $F_{M t}=F_{c e}+F_{p e}$. The contractile element force is the product of the force-length $F_{l}$ and the force-velocity $F_{v}$ relationships and the activation

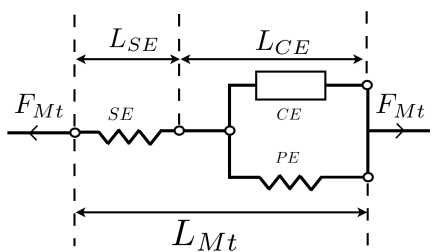

Fig. 6. Hill type musculotendon model

act, as follows:

$$
F_{c e}=a c t . F_{l} \cdot F_{v} .
$$

Note that $a c t_{\min }<a c t<1$ by structure of the model. The retained $F_{l}$ relation is $F_{l}=\exp \left(-\left(\left(L_{c e}-L_{c e_{0}}\right) /\left(L_{c e_{s h}}\right)\right)^{2}\right)$, where $L_{c e_{0}}$ is the optimal muscle fiber length and $L_{c e_{s h}}$ a constant shape parameter. The Hill force-velocity relation can be expressed as follows:

$$
F_{v}= \begin{cases}0 & V_{c e}<-V_{c e_{\max }} \\ \frac{b+a V_{c e}}{b-V_{c e}} & -V_{c e_{\max }}<V_{c e} \leq 0 \\ \frac{b+V_{c e}\left(F_{\left.v_{\max }+\left(F_{v_{\max }}-1\right) a\right)}^{b+V_{c e}}\right.}{\frac{V_{c e}}{V}>0}\end{cases}
$$

where $a, b$ et $F_{v_{\max }}$ are constant parameters, $V_{c e}$ is the contraction velocity of muscle fiber. The maximum velocity contraction of the muscle fiber is commonly taken as $V_{c e_{\max }}=10 . L_{c e_{0}}$. The force generated by the PE is given by : $F_{p e}=\left(e^{-\frac{p e_{s h}}{p e_{x \rightarrow m}\left(l_{c e}-1\right)}-1}\right) /\left(e^{p e_{s h}}-1\right)$, where $p e_{s h}$ and $p e_{x m}$ are constant parameters. Regarding the tendon stiffness, it can be approximated by a linear spring as shown in Zajac [20].

\section{Muscle redundancy and torque feedback}

A human joint is actuated by a pair of agonist-antagonist muscle group. Therefore muscle coactivation needs to be addressed. Previous human experimentation on sinusoidal standing perturbations [21] have shown a very small coactivation between the agonist and antagonist muscle group. Therefore, the coactivation will be neglected in our modeling.

The total joint net torque in our modeling is the following:

$$
\Gamma=r_{a} F_{M t_{a}}-r_{p} F_{M t_{p}}
$$

where $r_{a}$ and $r_{p}$ are the constant lever arm (since a double pulley model is used), $F_{M t_{a}}$ and $F_{M t_{p}}$ are the agonist and antagonist force developed by the muscles.

In human, a muscle force feedback is given by the Golgi Tendon Organs (GTO), since the GTO is in serial with the tendon, the gain $K_{G T O}$ is known to be constant. Since there is no coactivation in our model and since the lever arms are constants, the GTO feedback is the product of each muscle force with muscle lever arm.

Finally, in order to address muscle redundancy at the joint level (for a muscle group), we use the control scheme based on [22] depicted on Fig. 7. A torque feedback is given by Golgi Tendon Organs. The pulling muscle selection is based on the sign of the torque error. $a_{a}$ and $a_{p}$ are the agonist and antagonist activation signals which appear in Eq. (2); they are 
saturated to $1 .\left(Q^{T}\right)^{+}$is the transformation matrix from the joint-space to muscle space and $\Gamma_{d}$ the desired joint torque which is an image of an higher level supra-spinal control variable $U_{s s}$ since $K_{H}$ is a homogeneity gain fixed to 1 . The muscle model activation must be positive and since $\left(Q^{T}\right)^{+}$ is constant, the selection of the pulling muscle is based on the sign of the torque error.

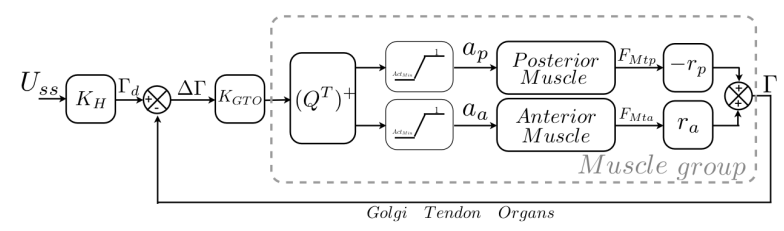

Fig. 7. Block diagram of the muscle redundancy management.

\section{Spindle reflex feedback}

In this study, the restraining reflex joint torques are considered in the model in order to limit the joint motion and to improve the fit with human joint positions. In human, the contraction velocity and the length of the musculotendon system are given respectively by $I I$ and $I a$ spindles sensors. It's well known that spindle feedback reflexes (SFR) are important in postural muscles, especially in terms of stiffness control and disturbance rejection. Many spindle models exist, at different level of physiology description, e.g. [23], but generally they are modelled with a pure gain and a small time delay [17] (35ms). Because SFR time delay is small and since no other time delays are introduced in our model, there will be neglected. Future works will introduce a nonlinear spindle model with time delay, in order to have a better physiological description.

Spindle sensors are located in parallel with muscle fibers (CE element), so their length change with $L_{c e}$ length. Then CNS adapt the SFR gains, in order keep a good measure sensitivity. In addition, the point-equilibrium-theory in motorcontrol literature, proposed by Feldman [24], argues that the CNS modulates the SFR gains to control the muscle stiffness and hence the joint position. This theory is the target of a vivid debate but there is converging evidence. Therefore, we will tune the SFR gains in section IV-A.

\section{E. Closed-loop modeling}

The CNS needs to manage redundant sets of actuators and sensors to perform the tracking task. Thus postural control can be assumed as an optimal control problem for the CNS. The Bardy et al.'s paradigm is composed at least of two main tasks: a standing task and a tracking task.

Firstly, a proportional-derivative $(P D)$ controller, in the operational space, is chosen as neural controller model to perform the tracking task. Since Masani et al. [25] argues that $P D$ controller is a good approximation of the control strategy in human standing, specially for the robustness on time delay (which will be the next step of our work). The actual tracking task sets only the horizontal head position ( $X$ axis in Fig. 5), so the actuated system is redundant with respect to the task. Therefore we use a jacobian pseudoinverse matrix to address the joint control vector. It is well attested that the use of the pseudoinverse matrix in kinematics redundant problems minimizes the norm of the velocity vector $\|\dot{\theta}\|^{2}$ at a given time. By analogy with inverse kinematics, the pseudoinverse matrix used in the control scheme depicted on Fig. 8 minimizes the norm of the supraspinal vector $\left\|U_{s s}(t)\right\|^{2}$. Then our model behaves like an optimal controller.

Secondly, standing must be guaranteed, that is the DIP must stay relatively close to the upright position and the CoP inside the base of support. This equilibrium constraint is managed by the SFR loops which constrain muscle lengths (i.e. joint positions) close to rest positions. In other words, it means that the vertical position of the head ( $Y$ axis in Fig. 5 ) is virtually bounded. Consequently, the proposed closedloop model scheme minimises an energetic criterion while minimising the joint deviations from rest position.

Therefore our non-linear control scheme is composed of a double inverted pendulum as biomechanical model, a muscle group (see section III-C), a classical PD controller in operational space, a torque feedback representing the GTO and joint positions and velocities feedback representing the SFR, as shown on Fig. 8.

On Fig. 8, $D K M$ is the direct kinematics model, $J^{+}$is the pseudoinverse matrix and $K_{I a}$ and $K_{I I}$ respectively the position and velocity feedback gains at spindles level.

\section{IDENTIFICATION RESULTS}

\section{A. Identification of equivalent spindle feedback gains}

Based on motor-control literature and as argued in section III-D, we propose to identify for each frequency step the SFR gains, i.e. $K I I_{1}, K I a_{1}, K I I_{2}$ and $K I a_{2}$, where index 1 stands for ankle and index 2 for hip. All other model parameters are taken constant and known prior to identification, chosen according to physiological considerations. They correspond to the same typical subject described in section II. In addition, the operational space gains are taken as $P=800$ and $D=1000$, in order to let the closed-loop model follow the desired head position with a good accuracy for all frequencies. As regarding physiological meanings, this tuning process is equivalent to adjusting as close as possible the dynamical reflex behaviors of each movement at a given frequency, to the actual ones.

In this study, SFR gains identification is addressed in the stochastic framework, where the maximum-likelihood approach makes it possible to derive a criterion to be optimized to estimate these gains and an asymptotic uncertainty domain associated with the estimated gains [26]. Under the usual Gaussian assumptions for the probability density function of the data and measurement errors, the maximum-likelihood estimator boils down to the least-square estimator, which minimizes the quadratic norm of the following output error:

$$
J=\sum_{i=1}^{2} \sum_{t=0}^{10 T}\left(\theta_{i_{h u m}}-\theta_{i_{\text {sim }}}\right)^{2}
$$




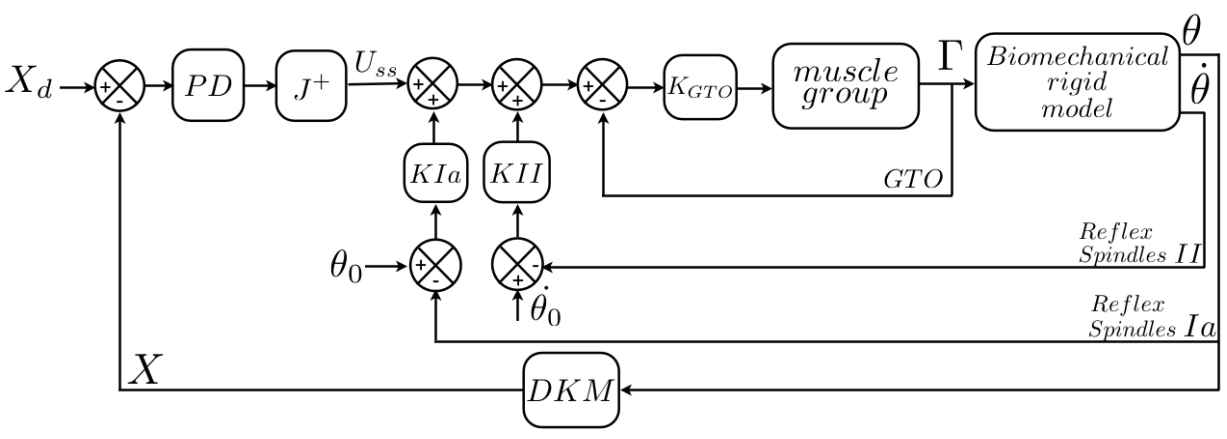

Fig. 8. General block diagram of the musculoskeletal postural coordination model. Input $X_{d}$ is the prescribed head position on $X$ axis, which is measured onto the subject. $P D$ represent a neural controller to track the target, $U_{s s}$ is the supraspinal neural signal, the output is the joint position $\theta$. The reflex loops on joint positions and velocities (provided via spindles) and on torque (GTO) change the $U_{s s}$ control signal, and hence modify muscle stiffness. The muscle group block is described on Fig. 7. $\theta_{0}$ and $\dot{\theta}_{0}$ are quiet stance values, $\theta_{=}(\pi / 2,0)^{T}$ and $\dot{\theta}_{0}=(0,0)^{T}$.

TABLE I

IDENTIFIED SPINDLE GAINS AND STANDARD DEVIATION

\begin{tabular}{|l|l|l|l|l|}
\hline f (Hz) & $K I I_{1}($ u.SI) & $K I a_{1}$ (u.SI) & $K I I_{2}($ u.SI & $K I a_{2}($ u.SI) \\
\hline 0.2 & $1815 \pm 0.01$ & $553.4 \pm 9 \mathrm{e}-5$ & $132.7 \pm 0.02$ & $190.8 \pm 0.04$ \\
\hline 0.4 & $1987 \pm 44.9$ & $257.4 \pm 0.56$ & $185.6 \pm 16.4$ & $1.003 \pm 0.57$ \\
\hline 0.65 & $1203 \pm 8.7$ & $499.9 \pm 0.16$ & $21.92 \pm 0.55$ & $4.990 \pm 0.15$ \\
\hline
\end{tabular}

where $T$ is signal period for a given frequency, $\theta_{\text {hum }}$ and $\theta_{\text {sim }}$ are respectively the human experimental and simulation output joint positions. Because the model employed is nonlinear in its parameters, the minimization is usually performed by iterative search. We used here a Gauss-Newton algorithm to minimize eq. (4). The asymptotic standard deviation associated with the estimated parameters are taken as the square root of the diagonal entry of the inverse of Fisher information matrix [26]. Both estimated SFR gains and associated standard deviations are given in table I, for $f=0.2 \mathrm{~Hz}, f=0.4 \mathrm{~Hz}$ and $f=0.65 \mathrm{~Hz}$.

\section{B. Comparison of experimental data with identified model results}

In order to evaluate the quality of our model, hence to give indications about the validity of our modelling and identification results, we will compare here the identified model simulation results and actual data. Fig. 9, 10 and 11 show the actual human movement of the head, ankle and hip joints, the CoP location and the ones obtained by the identified model parameters. The identified model simulation joint angles results for in-phase and anti-phase modes and during the phase transition are similar to the ones measured experimentally on human being, as it obviously should be since the criterion 4 minimizes the quadratic norm of modeldata joint angles discrepancy. Moreover, it is worth noting that the CoP location obtained by our identified model is very close to actual one. This evidences the good quality and relevance of our modelling. Indeed, since CoP location is connected to actuation torques, then such a small simulation - actual data discrepancy indicates that the actuation torques needed by our virtual human to achieve the tracking task should be quite similar to actual ones. In addition, since simulated CoP remains within the base of support, the equilibrium constraint is satisfied.

\section{Hysteresis phenomenon}

A further model validation can be assessed by analysing the abilities of our model to exhibit the hysteresis phenomenon, hallmark of non-linear systems, which has been observed in human experiments [11]. Note also, that this hysteresis phenomenon has never been modeled in the postural coordination framework. When the target frequency is up-chirped and then down-chirped, our closed loop model, with SFR gains taken as the mean values of the previous identified gains $K I I 1=1500, K I a 1=300, K I I 2=150$, $K I a 2=10$, exhibits the hysteresis phenomenon. Note that the gain values of the controller and the dynamics of the reference target influence the hysteresis region. Current work now examines the energetic cost for different types of reference dynamics around the transition frequency in order to better understand the hysteresis phenomenon.

\section{CONCLUSiOnS}

The musculoskeletal closed-loop model we have developed provides realistic predictions of postural sway movements during head tracking task. The closed-loop modeling also allows to reproduce the hysteresis phenomenon observed in human experiments. Spindle reflex feedback loops gain were successfully estimated using actual data, it remains to study further their variation with frequency for all subjects involved in our experiments. Nevertheless, we believe that our model of postural coordination is promising in capturing behavioral invariants observed in human postures.

More detailed sensory dynamics, as non-linear spindles, in addition to the time delay are under development to be considered in the model. The time delay and small modifications of muscle dynamics should allow our model to be applied on individual with stroke. Finally, to obtain a more realistic model, we will add more muscle actuators. But then, sensory information fusion and redundancy resolution will become an issue. 

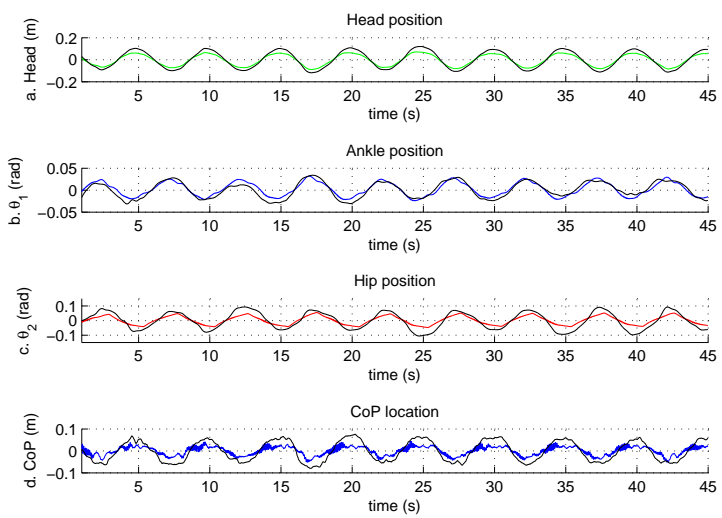

Fig. 9. Identified model simulation results (color) and actual data (black line). $f=0.2 \mathrm{~Hz}$
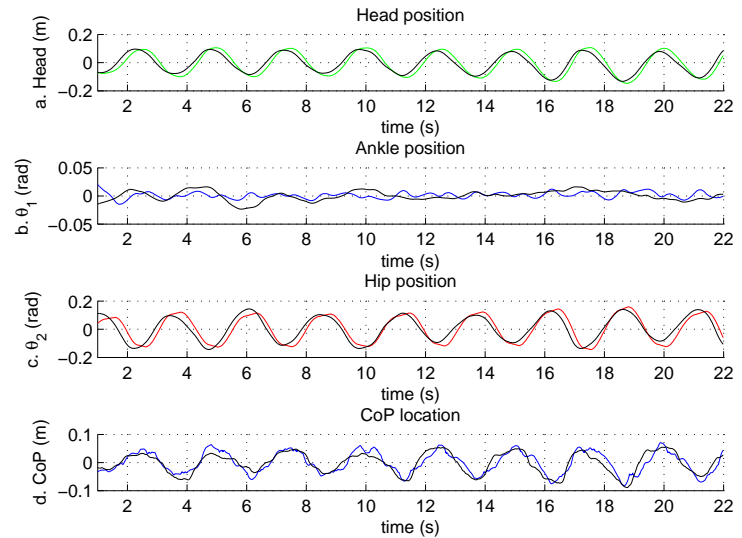

Fig. 10. Identified model simulation results (color) and actual data (black line). $f=0.4 \mathrm{~Hz}$
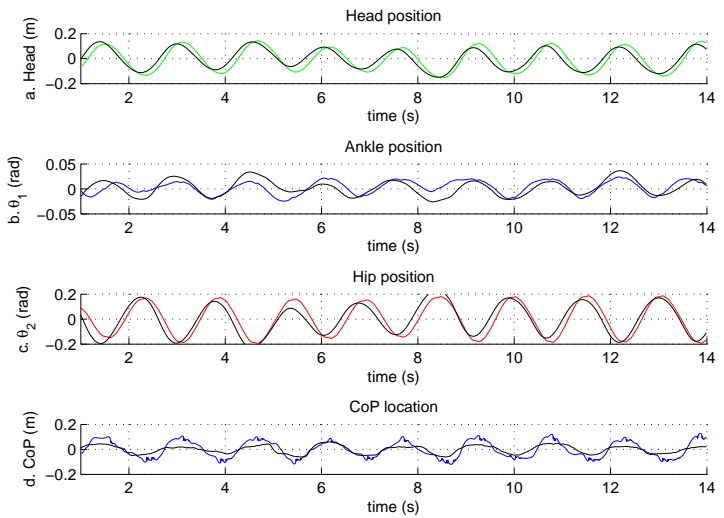

Fig. 11. Identified model simulation results (color) and actual data (black line). $f=0.65 \mathrm{~Hz}$

\section{REFERENCES}

[1] B. Bardy, O. Ouillier, R. Bootsma, and T. Stoffregen, "Dynamics of human postural transitions," ExP. Psychol. Hum. Percept. Perform., vol. 28, pp. 499-514, 2002.

[2] J. Kelso, Dynamics Patterns, The Self-Organization of brain and behavior. The MIT Press, 1995.

[3] L. Martin, V. Cahouet, M. Ferry, and F. Fouque, "Optimization model predictions for postural coordination modes," Journal of Biomechanics, vol. 39, pp. 170-176, 2006.

[4] V. Bonnet, J. Lagarde, P. Fraisse, N. Ramdani, S. Ramdani, P. Poignet, and B. Bardy, "Modelling of the human postural coordination to improve the humanoids control of balance," IEEE Int. Conf. on Humanoid Robots, 2007.

[5] H. Haken, J. Kelso, and H. Bunz, "A theorical model of phase transitions in human hand movements," Biological Cybernetics, vol. 51, pp. 347-356, 1985.

[6] L. Nashner and G. McCollum, "The organization of postural movements: a formal basis ansd experimental synthesis," Behavioral and Brain Sciences, vol. 26, pp. 135-172, 1985.

[7] H. Hemami, F. Weimer, C. Robinson, C. Stockwell, and V. Cvetkovic, "Biped stability considerations with vestibular models," IEEE Trans. Autom. Contr., vol. AC-23, pp. 1074-1079, 1978.

[8] A. Kuo, "An optimal control model for analyzing human postural balance," IEEE Trans. Biomed. Eng., vol. 42, pp. 87-101, 1995.

[9] S. Park, F. Horak, and A. Kuo, "Postural feedback responses scale with biomechanical constraints in human standing," Exp. Brain. Res., vol. 154 , pp. 417-427, 2004

[10] S. Jo and S. G. Massaquoi, "A model of cerebellum stabilized and scheduled hybrid long-loop control of upright balance," Biological Cybernetics, vol. 91, no. 3, pp. 188-202, 2004.

[11] B. Bardy, L. Marin, T. Stoffregen, and R. Bootsma, "Postural coordination modes considered as emergent phenomena," Exp. Psychol. Hum. Percept. Perform., vol. 25, pp. 1284-1301, 1999.

[12] O. Oullier, B. Bardy, T. Stroffregen, and R. Bootsma, "Postural coordination in looking and tracking tasks," Human Movement Sciences, vol. 21, pp. 147-167, 2002.

[13] M. Ferry, "Formation de la trajectoire pour des taches supra-posturales: modélisation et experimentation," Ph.D. dissertation, Univ. Joseph Fourrier, Genoble I, 2004.

[14] C.F.Runge, C. Shupert, F. Horak, and F. Zajac, "Ankle and hip postural strategies defined by joint torques," Gait and Posture, vol. 10, pp. 161170, 1999.

[15] K. Barin, "Evaluation of a generalized model of human postural dynamics and control in the sagittal plane," Biological Cybernetics, vol. 61 , pp. 37-50, 1989

[16] V. Cahouet, L. Martin, and D. Amarantini, "Static optimal estimation of joint accelerations for inverse dynamics problem solution," Journal of Biomechanics, vol. 35, pp. 1507-1513, 2002.

[17] A. van Soest, W. Haenen, and L. Rozendaal, "Stability of bipedal stance: the contribution of cocontraction and spindle feedback," Bio logical Cybernetics, vol. 88, pp. 293-301, 2003.

[18] A. van Soest and L. Rozendaal, "The inverted pendulum model of bipedal standing cannot be stabilized through direct feedback of force and contractile element length and velocity at realistic series elastic element stiffness," Biological Cybernetics, vol. 99, pp. 29-41, 2008.

[19] R. Riener and T. Fuhr, "Patient-driven control of fes-supported standing up: a simulation study," IEEE Trans. in Rehabilitation Eng., vol. 6 , pp. 113-124, 1998.

[20] F. Zajac, "Muscle and tendon: properties; models, scaling, and application to biomechanics and motor control," Bourne JR (ed) Crit. Rev. Biomed. Eng., vol. 17, pp. 359-411, 1989.

[21] J. J. Buchanan and F. B. Horak, "Emergence of postural patterns as a function of vision and translation frequency," J. Neurophysiol., vol. 81, pp. 2325-2339, 1999.

[22] J. M. Winters and P. E. Crago, Biomechanics and Neural Control of Posture and Movement. Springer-Verlag, 2000.

[23] Z. Hasan, "A model of spindle afferent response to muscle stretch," J. Neurophysiol., vol. 49, pp. 989-1006, 1983.

[24] A. Feldman, "Functional tuning of the nervous system with control of movement or maintenance of a steady posture. mechanographic analysis of execution by man of the simplest motor tasks," Biophysics, vol. 11, pp. 766-775, 1966.

[25] K. Masani, A. Vette, and M. Popovic, "Controlling balance during quiet standing: Proportional and derivative controller generates preceding motor command to body sway position observed in experiments,' Gait and Posture, vol. 23, pp. 164-172, 2005.

[26] E. Walter and L. Pronzato, Identification of Parametric Models from Experimental Data. Springer-Verlag, 1997. 